\title{
CUARENTA AÑOS DESPUES DE ALMA ATA: LA ACTUALIDAD DE SUS CONCEPTOS FUNDAMENTALES
}

\author{
David Tejada de Rivero 1,a,", Temístocles Sánchez ${ }^{1, b}$, José Ormachea 1,c, Fernando Carbone-Campoverde ${ }^{2, \mathrm{~d}}$ \\ RESUMEN \\ Al conmemorarse 40 años de la Conferencia Internacional de Atención Primaria de Salud de 1978 en Alma Ata (Kazajistán, \\ ex URSS), la vigencia de sus principios fundamentales se mantiene, siendo importante recordarlos como un referente para el \\ futuro mediato, y proyectarlos a la salud para todos, pendiente de construir con todos.
}

Palabras Clave: Alma Ata; Atención Primaria de la Salud; Cuidado Integral de la Salud; Salud para Todos. (Fuente: DeCS BIREME).

FORTY YEARS AFTER ALMA-ATA: CURRENCY OF ITS FUNDAMENTAL CONCEPTS

\begin{abstract}
As we commemorate 40 years of the International Conference of Primary Health Care celebrated in Alma-Ata (Kazakhstan, ex-USSR) in 1978, the currency of its fundamental principles holds strong, and it is important to remember them as a referent for the near future, and to project them to every individual's health, to be built by all.
\end{abstract}

Keywords: Alma Ata, Comprehensive Healthcare, Health for all, Primary Health Care. (source: MeSH NLM).

\section{INTRODUCCIÓN}

No es la primera vez que escribimos sobre Alma Ata, David Tejada de Rivero (Figura 1), en el 2003 por la celebración de 25 años de la Conferencia Internacional de Salud de 1978 y a solicitud de la doctora Mirtha Roses Periago, entonces directora de la Organización Panamericana de la Salud (OPS), fue invitado a hacer un relato ${ }^{(1)}$ de todos los procesos técnicos y políticos que se dieron antes de la misma hasta su realización en Alma Ata.

Esta conferencia -de Alma Ata- ha sido sin duda el evento de política de salud internacional más importante en la década de los 70 y marcó un hito en la salud en el mundo. Asistieron a la misma 134 países, 67 organismos internacionales y más de un centenar de organizaciones no gubernamentales. Debemos destacar que la declaración final de la conferencia (2) se alcanzó por consenso y se aprobó por unanimidad. Adicionalmente hubo recomendaciones recogidas de todos y cada uno de los países en un segundo documento (2). El proceso previo fue una construcción descentralizada y participativa (18 borradores, intercambiados sucesivamente por fax y/o correo postal) a lo largo de dos años, y que llegó a involucrar a los países de todas las regiones del mundo.

\section{ALMA ATA EN CONTEXTO Y PERSPECTIVA}

Es imprescindible recordar que a mediados del siglo pasado la Organización Mundial de la Salud (OMS), dirigida entonces por el Dr. Halfdan T. Mahler, se propuso rescatar y valorar algunas experiencias en el mundo, principalmente de China con su medicina tradicional y otras formas académicas no occidentales. Se rescataron experiencias en la India, África y América Latina, donde se pudo superar las inequidades e injusticias en la salud. La OMS publicó en ese entonces un libro con estas y otras experiencias. El mayor mérito de la OMS fue darles un reconocimiento científico a las mismas a pesar de las duras críticas y oposiciones que persistieron hasta la víspera de la Conferencia de Alma Ata.

Los borradores y originales a discutirse en Alma Ata plantearon finalmente en esa época el término «Primary

\footnotetext{
Asociación de Consultores Internacionales en Salud y Desarrollo Social. Lima, Perú.

Medicus Mundi Navarra Aragón Madrid. España.

Médico, magister en Salud Pública; especialista en planificación y desarrollo; especialista en ciencias políticas; ${ }^{\mathrm{b}}$ Médico. Doctor en Salud Pública; ${ }^{\mathrm{c}}$ Médico, especialista en Administración de Hospitales, especialista en Salud Pública; ${ }^{\mathrm{d}}$ Médico, magister en Gestión de Centros y Servicios de Salud.

* Fallecido el 04/11/2018

Recibido: 03/09/2018 Aprobado: 05/12/2018 En línea: 21/12/2018
}

Citar como: Tejada de Rivero D, Sánchez T, Ormachea J, Carbone-Campoverde F. Cuarenta años despues de Alma Ata: la actualidad de sus conceptos fundamentales. Rev Peru Med Exp Salud Publica. 2018;35(4):675-7. doi: 10.17843/rpmesp.2018.354.3902. 


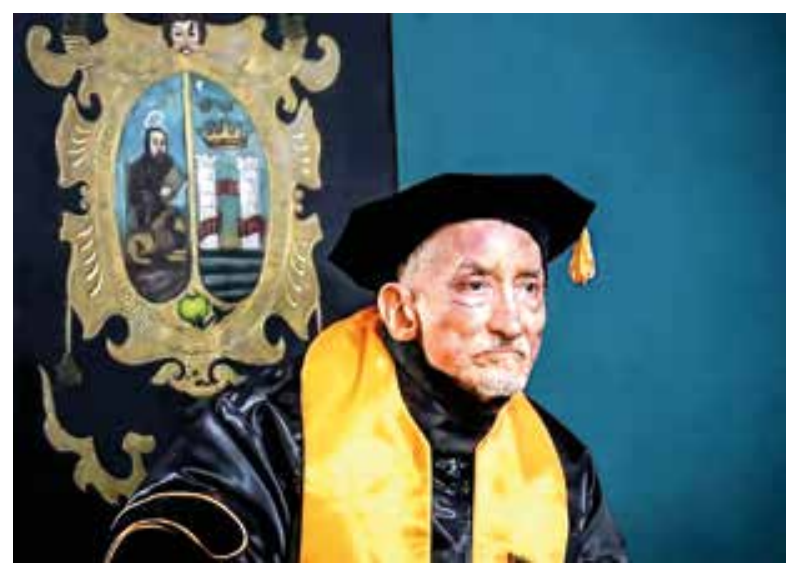

Figura 1. Doctor David Tejada de Rivero, durante su investidura de Doctor Honoris Causa en la Universidad Nacional Mayor de San Marcos.

Health Care», cuya traducción a otros idiomas desvirtúo lamentablemente el significado de esos términos. El término original (Care) se refería a «cuidado» y no a «atención». El término "Primary» fue mal interpretado. La idea de Alma Ata era considerar la palabra «Primary» como lo esencial, nuclear, fundamental, sustantivo y prioritario. Pero se interpretó después en su sentido opuesto, como un servicio, todo médico reparativo y barato para atender a los pobres. Es muy claro en la «Declaración de Alma Ata» lo esencial se refiere a «cuidado de la salud» y no a una «atención médica reparativa orientada a la enfermedad» ${ }^{(1-3)}$.

Es necesario recordar que la idea de la Conferencia de Alma Ata tendría que haber sido la respuesta concreta a la meta social de «Salud para todos en año 2000». Esta meta también ha sido desvirtuada porque nunca se pensó que en el año 2000 los países del mundo superarían totalmente las injusticias e inequidades en salud. La idea central de «salud para todos en el año 2000», era que para dicho año todos los países del mundo tendrían «Políticas Públicas de Estado» orientadas a este fin. Vale la pena aclarar que se trataba de políticas a largo plazo, obligatorias para todos y no circunscritas a efímeros periodos presidenciales de gobierno ${ }^{(1,3)}$.

Lamentablemente en la actualidad no se han superado las inequidades existentes en la década de los 70 del siglo pasado; y, en algunos casos, las injusticias e inequidades en los niveles de salud se han incrementado. Uno de los factores que más ha pesado en ello, ha sido el persistir en una atención médica reparativa orientada a la enfermedad y no en actuar sobre los determinantes sociales de la salud presentes en todos los sectores y ámbitos de los Estados y en poblaciones nacionales ${ }^{(1,3,4)}$.

En el mundo globalizado de hoy, y con la amenaza de una pérdida creciente de las soberanías nacionales, una de las pocas medidas que existen para tener control sobre el destino de un país es la construcción de democracias auténticas, descentralizadas y participativas. Hoy es imprescindible transferir, mejor dicho «devolver» el poder político para tomar las decisiones que afectan a los países a su punto de origen, que es la ciudadanía.

Alma Ata no ha sido sólo la teorización de experiencias recogidas el año de su realización. La Conferencia de Alma Ata ha inspirado nuevos intentos de construir de forma innovadora y participativa, la salud para todos y por todos.

Finalmente debemos reconocer todos que en 40 años el mundo ha cambiado en muchos aspectos. Un ejemplo de ello es el impacto climático del calentamiento global, la pérdida de los glaciares y de las tierras cultivables, que afectan a su vez las condiciones saludables del clima, y cimientan, según algunos estudiosos, la destrucción definitiva del mundo. Paralelamente los Gobiernos y los Estados no acatan medidas de prevención frente a estos hechos; no obstante, los acuerdos formales que los países toman al respecto a escala mundial. Es como si este determinante social de la salud (y de la vida humana sin duda alguna) importase menos que los beneficios económicos que se derivan de las actividades económicas que afectan el clima y la naturaleza.

Nuevos factores, y nuevas condiciones refuerzan la necesidad de actualizar y reforzar las metas originales de Alma Ata. La conmemoración de los 40 años de la gran Conferencia Internacional de Salud de 1978 es una oportunidad para que la OPS asuma el liderazgo de promover y conducir un proceso participativo y descentralizado para culminar en -un nuevo Alma Ata-, una Conferencia de Salud de las Américas que luego propicie una nueva conferencia internacional de alcance mundial, que no puedan ser desvirtuadas ni distorsionadas por el facilismo de los sectores técnicos y del mercantilismo de algunas sociedades nacionales.

\section{CONCLUSIONES}

Una conferencia que pretenda alcanzar los fines propuestos, debe partir del principio que la salud depende de todos y por tanto «El Cuidado Integral de la Salud por Todos y para Todos» debe estar en todas las políticas.

Lo anterior requiere que todos los sectores económicos y sociales, y consecuentemente los Ministerios y dependencias respectivas, incluyan a la salud en sus políticas de forma concreta y dentro de un marco referencial, integral de tipo nacional (Política Nacional de Salud).

Todos los sectores del Estado deben incluir en su accionar planes y actividades dirigidas a apoyar el trabajo en salud liderado por los Ministerios de Salud de los países, los cuales pasarían a atender responsabilidades fundamentales en la promoción, coordinación, acompañamiento y monitoreo de 
una política multisectorial de salud. Coincidiendo esto con el enfoque de los objetivos de desarrollo sostenible ${ }^{(5)}$.

Contribución de los autores: todos los autores participaron de forma conjunta en el artículo.
Fuentes de financiamiento: autofinanciado.

Conflictos de interés: declaramos que no existe relación, condición o circunstancia que pueda afectar la objetividad en la interpretación del artículo; ni económica ni institucional.

\section{REFERENCIAS BIBLIOGRÁFICAS}

1. Tejada de Rivero DA. Alma-Ata: 25 años después. Revista Perspectivas de Salud. OPS. 2003;8(2)3-7.

2. World Health Organization. Atención Primaria de Salud. Informe de la Conferencia Internacional sobre Atención Primaria de Salud. Geneva: WHO;1978.

3. Tejada de Rivero DA. Lo que es la atención primaria de la salud: algunas consideraciones a casi treinta y cinco años de Alma-Ata. Rev Peru Med Exp Salud Publica. 2013;30(2):283-7.

4. Organización Mundial de la Salud OMS. Salud en Todas las Politicas. 2015.

5. PNUD. Objetivos de Desarrollo Sostenible [acceso 30 nov 2018] Disponible en : http://www.undp.org/content/undp/es/ home/sustainable-development-goals.html

Correspondencia. Fernando Carbone Campoverde Dirección: Calle Ing. Luis Montero 198, oficina 203, San Borja, Lima, Perú;

Teléfonos 4761842

Correo electrónico: fernando.carbone@medicusmundi.pe

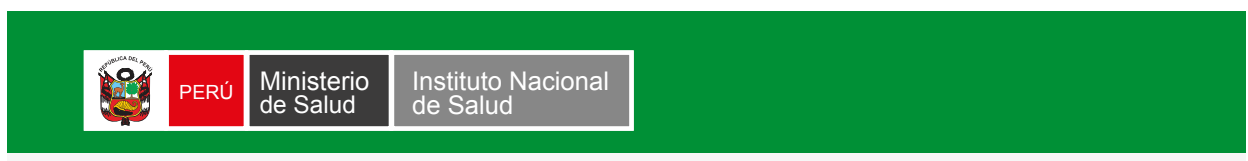

Inclusión social en salud: aporte de las tecnologías para el tratamiento de enfermedades desatendidas

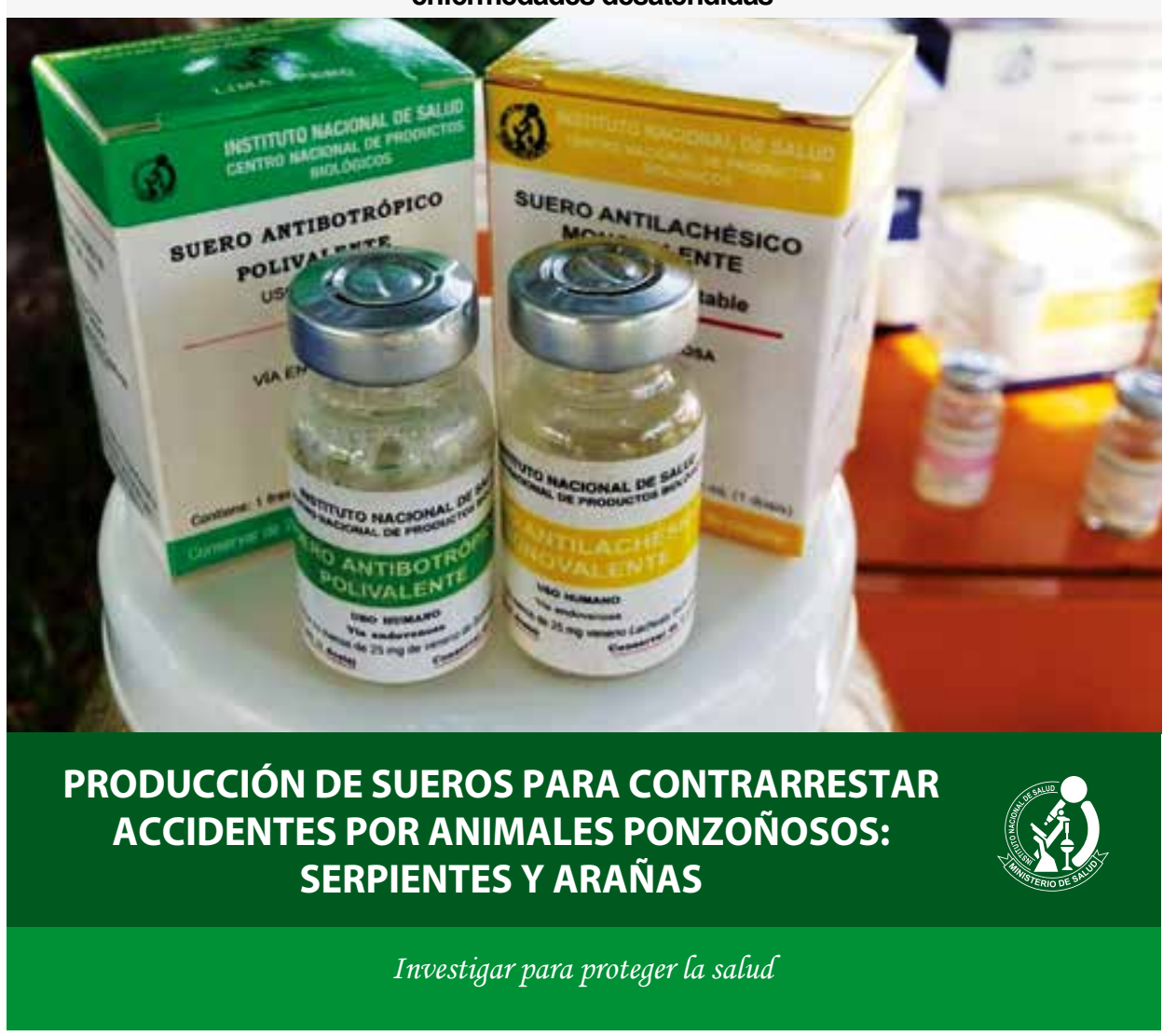

